\title{
How to improve organisational staff support? Suggestions from humanitarian workers in South Sudan
}

\author{
Hannah Strohmeier ${ }^{1,2,3}$, Willem F. Scholte ${ }^{3,4,5}$ \& Alastair Ager ${ }^{2,6}$ \\ ${ }^{1}$ MSc, MA, Department of Anthropology, Yale University, New Haven, Connecticut, USA, ${ }^{2}$ Institute for Global Health and Development, \\ Queen University, Edinburgh, UK, ${ }^{3}$ Antares Foundation, Amsterdam, The Netherlands, ${ }^{4} \mathrm{MD}$, PhD, Equator Foundation, Diemen, The Netherlands, \\ ${ }^{5}$ Amsterdam UMC, Department of Psychiatry, University of Amsterdam, Amsterdam, The Netherlands, ${ }^{6} \mathrm{PhD}$, Institute for Global Health and Development, \\ Queen Margaret University, Edinburgh, UK, ${ }^{6}$ Mailman School of Public Health, Columbia University, New York, USA
}

\section{Abstract}

Humanitarian workers experience high symptom burdens of common mental health problems. This requires action from the organisations they are employed with. However, many studies have documented continuing weaknesses in organisational staff support, as well as disparities in access to the services for national and international staff. Systematic data capturing suggestions from humanitarian workers on how to tackle this situation within a specific crisis setting is rarely available. This study addresses this gap through qualitative content analysis of the suggestions from the 210 humanitarian workers based in South Sudan collected through an online survey in 2017. Five major themes emerged regarding proposed improvements: 'Competitive benefit and salary packages'; 'internal work climate and organisational culture'; 'equality within and between organisations'; 'skill enhancement and personal development' and 'physical safety and security'. For both national and international staff, improved access to psychosocial support services was the most frequent proposal. Apart from this suggestion, their top priorities for improvement of staff support differed greatly. National staff emphasised improvements related to training and greater equality between employees. International staff emphasised improvements related to time off and team cohesion. Findings provide a clear case for organisations to assess their services and offer a potential framework to inform future interventions that better address the priorities of the humanitarian community as a whole.

Keywords: Humanitarian workers, international staff, national staff, South Sudan, staff support

\section{INTRODUCTION}

\section{Background}

Humanitarian work is associated with an elevated risk of traumatic event and chronic stress exposure. Although some humanitarian workers cope with life in crisis situations without developing prolonged symptoms of mental ill-health (McKay, 2011), research confirms that common mental health problems, such as post-traumatic stress disorder (PTSD), depression, anxiety, burnout and hazardous alcohol consumption, are widespread among this occupation group (e.g. Ager, Pasha, Yu, Duke, Eriksson, \& Cardozo, 2012; Eriksson et al., 2012; Lopes Cardozo et al., 2005; Lopes Cardozo et al., 2012; Lopes Cardozo et al., 2013; Shah, Garland, \& Katz, 2007). This can have

\begin{tabular}{|l|l|}
\hline \multicolumn{2}{|c|}{ Access this article online } \\
\hline Quick Response Code: & Website: \\
& www.interventionjournal.org \\
& \\
&
\end{tabular}

severe implications for the personal and professional lives of humanitarian workers. Examples of consequences on life course of PTSD are significantly elevated risks for marital instability and unemployment (Brunello et al., 2001). Experiencing mental health problems can also impact the organisations humanitarian workers are employed with: 'Stressed out' employees have overall higher accident and illness rates, are absent from work and consult health services more frequently, and are less

Address for correspondence: Ms. Hannah Strohmeier, MSc, MA, Queen Margaret University, Edinburgh, EH21 6UU, UK.

E-mail: hstrohmeier@qmu.ac.uk

This is an open access journal, and articles are distributed under the terms of the Creative Commons Attribution-NonCommercial-ShareAlike 4.0 License, which allows others to remix, tweak, and build upon the work non-commercially, as long as appropriate credit is given and the new creations are licensed under the identical terms.

For reprints contact: reprints@medknow.com

How to cite this article: Strohmeier, H., Scholte, W.F., \& Ager, A. (2019). How to improve organisational staff support? Suggestions from humanitarian workers in South Sudan. Intervention, 17(1), $40-49$. 
Strohmeier et al.: How to improve organisational staff support?

efficient and effective in carrying out their duties (Antares Foundation, 2012; Curling \& Simmons, 2010). Apart from individual awareness and self-care, preventing and managing the mental health of humanitarian workers thus require staff support provided by organisations on moral, legal (duty of care, due diligence) and economic grounds (Antares Foundation, 2012; Curling \& Simmons, 2010; Porter \& Emmens, 2009). Such support is considered an important determinant of the mental health of humanitarian workers (Strohmeier \& Scholte, 2015), and efficacy is greatest when services match the specific context on the ground (Care for the caretakers, 2010; Gilbert, 2016; Kahn, 2018; Omidian \& Panter-Brick, 2015; Pigni, 2014).

The need for tailor-made, systematic staff support is widely recognised in the humanitarian community. Entities concerned with aid worker wellbeing developed guidelines and key actions for organisations towards this end (Antares Foundation, 2012; IASC, 2007; Porter \& Emmens, 2009), and much progress has been achieved in recent years (Welton-Mitchell, 2013). Nevertheless, surveys and evaluations show that staff support systems are by and large still underdeveloped and the services offered oftentimes underfunded and insufficient (Gilbert, 2016; Surya, Jaff, Stilwell, \& Schubert, 2017; Welton-Mitchell, 2013). Further, there is a huge inconsistency between staff support services across and within organisations, especially with regards to the distinct needs of national and international staff and their access to services, respectively (Porter \& Emmens, 2009; Stoddard, Harmer, \& Haver, 2011). Systematically collected and analysed data capturing suggestions from humanitarian workers on how to tackle this situation within a specific crisis setting are not widely available. This is concerning given that it is these very people who experience life in crises and whose mental health is at stake.

Our study addresses this gap through a focus on suggestions from humanitarian workers based in South Sudan collected through an online survey in 2017. Specifically, we apply qualitative content analysis to explore the responses of national and international staff to the question how organisations can improve their staff support services. We begin by contextualising our study with an overview of the crisis setting South Sudan. Next we elaborate on the methods and proceeding of our study, followed by the presentation of results. We discuss these with a focus on similarities and differences regarding the most frequently delivered suggestions by national and international staff, and recommendations for action. We conclude with overall implications for practice and future research.

\section{Context}

South Sudan is the youngest state in the world and one of the four countries globally where the humanitarian situation is worse than ever (UN News, 2017). Armed conflict, inter-communal violence, disease outbreaks, climatic shocks and economic stress have left seven million people (more than half of the population in the country) in need of humanitarian assistance and protection. The multiplicity of crises in South Sudan has led one in three people to flee their home, causing the largest refugee crisis in Africa crisis (United Nations High Commissioner for Refugees [UNHCR], n.d.; United Nations Office for the Coordination of Humanitarian Affairs [UNOCHA] South Sudan, 2017).

The violent chaos in South Sudan affects humanitarian workers too. This includes obstacles related to aid delivery as well as problems regarding their own safety: More than 30 major attacks against civilian aid operations in 2015 and more than 50 in 2016 made South Sudan the most dangerous country for aid workers globally in both years (Humanitarian Outcomes, 2016, 2017). Largely a consequence of shootings and bodily assaults, South Sudan also claimed the highest number of aid worker victims and deaths in 2016. The outbreak of violence in the country's capital of Juba in July that same year reached a level of severity that caused many embassies and aid agencies to temporarily evacuate large parts of their international workforce (Deutsche Welle, 2016). The United Nations (UN) classifies the duty station of South Sudan as category 'E' (the highest grade of difficulty regarding the conditions of work and life) and as 'Non-family Duty Station' (dependents are not allowed to be present for more than six months due to security and safety reasons) (United Nations International Civil Service Commission [ICSC], 2016, 2017; United Nations Office of Human Resource Management [UNOHRM], n.d.).

Some organisations, especially UN entities, grant specific monetary allowances in response to the challenges attached to South Sudan. Examples are the 'Non-family Service Allowance' and 'Hardship Allowance'. Other typical benefits the UN and some non-governmental organisations (NGOs) grant are 'Rest and Recuperation' (R\&R) (additional, paid days of leave) and special safety and security measures, such as evacuation in case of need. However, if and to whom these and other services are available depends on the respective organisation. As elsewhere, staff support in South Sudan differs considerably between the organisations and national and international staff, whereby services available to national staff are oftentimes restricted (ICSC, 2016; Stoddard et al., 2011).

\section{Methodology}

\section{Study design and ethical clearance}

We collected the data as part of a larger cross-sectional online survey on the mental health and wellbeing of humanitarian workers in South Sudan. This survey was undertaken in 2017 and provided participants with the opportunity to share 'any suggestions how humanitarian organisations can improve their staff support services'.

The survey was conducted in English, the working language of South Sudan. It was accessible for one month and took approximately $45 \mathrm{~min}$ to complete. Queen Margaret University, Edinburgh, granted ethical clearance for the research project. Participants gave informed consent prior to their participation in the survey. 
Strohmeier et al.: How to improve organisational staff support?

\section{Eligibility criteria for participation}

We contacted 124 organisations that participated in the 2017 South Sudan Humanitarian Response Plan and/or the Humanitarian Country Team. We requested their support of the survey if they had operated in South Sudan for at least one year and employed a minimum of 10 staff in the country. Forty-five organisations - four UN entities, 20 international NGOs (INGOs) and 21 national NGOs (NNGOs) - confirmed their support. Eligible for participation were paid humanitarian workers (national and international staff, consultants and UN volunteers) from these organisations whose official duty station was located in South Sudan at the time of survey implementation.

\section{Data analysis}

We applied qualitative content analysis to examine participants' responses to the open-ended question how humanitarian organisations can improve their staff support services. The analysis followed an inductive approach (Elo \& Kyngas, 2007) and focused on manifest content, that is the literal, visible and obvious content of participants' written answers (Bengtsson, 2016; Graneheim \& Lundman, 2004).

The qualitative content analysis process comprised five key steps. First, we collated and read the answers of the participants multiple times to gain an in-depth overview of the data. Second, we extracted all content and divided it into meaning units, that is arrangements of words and sentences that convey the same principal meaning (Graneheim \& Lundman, 2004). Third, we formulated condensed meaning units. Fourth, we abstracted these condensed meaning units into sub-themes based on similarity in content, whereby each condensed meaning unit was assigned to one sub-theme only. Fifth, we merged these sub-themes into major themes. Following the understanding of Graneheim \& Lundman (2004), these themes reflect particular threads of meaning but are not mutually exclusive.

Using this coding frame as the basis, we added another layer of analysis by counting the meaning units assigned to each sub-theme and theme, respectively. We disaggregated these numbers by national and international staff - the two main employment categories in the humanitarian sector to facilitate presentation and discussion of the results in light of this common divide.

\section{RESULTS}

\section{Participants and structure of responses}

A total of 277 humanitarian workers participated in the online survey and 210 responded to the open-ended question on how organisations can improve their staff support services (based on the number of staff that had access to the survey as reported by organisations, a response rate in the order of $7 \%-8 \%)$. More than three-quarters $(78 \%)$ of these were male, and almost half (48\%) of the sample was between 30 and 39 years old. Slightly more humanitarian workers were based in Juba (54\%) than in the field (46\%), and about one-third (31\%) worked as manager/coordinator. Over half

\begin{tabular}{|c|c|}
\hline Variable & $\%$ \\
\hline \multicolumn{2}{|l|}{ Gender } \\
\hline Female & 22.4 \\
\hline Male & 77.6 \\
\hline \multicolumn{2}{|l|}{ Age } \\
\hline$<30$ & 20.0 \\
\hline $30-39$ & 48.1 \\
\hline $40-49$ & 25.2 \\
\hline $50+$ & 6.7 \\
\hline \multicolumn{2}{|l|}{ Level of education } \\
\hline Secondary school/high school & 7.1 \\
\hline Higher vocational education/technical training & 13.8 \\
\hline University (BA) & 43.3 \\
\hline Postgraduate (MA, MSc, PhD) & 35.7 \\
\hline \multicolumn{2}{|l|}{ Duty station } \\
\hline Capital (Juba) & 53.8 \\
\hline Field & 46.2 \\
\hline \multicolumn{2}{|l|}{ Job function } \\
\hline Administrative & 8.1 \\
\hline Human resources & 5.7 \\
\hline Logistics & 4.8 \\
\hline Technical/programme & 23.3 \\
\hline Manager/coordinator (programme or operations) & 31.0 \\
\hline Country director/head of mission & 7.1 \\
\hline \multicolumn{2}{|l|}{ Employment category } \\
\hline National staff & 57.6 \\
\hline International staff & 39.5 \\
\hline Consultant & 1.5 \\
\hline UN volunteer & 1.4 \\
\hline \multicolumn{2}{|l|}{ Organisation type } \\
\hline National NGO & 24.8 \\
\hline International NGO & 62.4 \\
\hline UN entity & 12.9 \\
\hline
\end{tabular}

of the participants identified themselves as national staff $(58 \%)$ and were employed by an INGO (62\%) [Table 1].

Participants' responses varied in length and scope. Most consisted of one to three sentences and captured one to two meaning units. Only few responses captured a wide range of suggestions and meaning units, respectively.

\section{Emerging themes}

The data analysis revealed five inter-related themes of suggestions on how organisations can improve their staff support services. Ordered by the overall highest share of meaning units constituting each theme these are 'Theme 1: Competitive benefit and salary packages' (50\%), 'Theme 2: Internal work climate and organisational culture' (18\%), 'Theme 3: Equality within and between organisations' (14\%), 'Theme 4: Skill enhancement and personal development' (12\%) and 'Theme 5: Physical safety and security' $(6 \%)$ [Figure 1].

\section{Theme 1: Competitive benefit and salary packages}

Ten sub-themes formed the theme 'Competitive benefit and salary packages' [Figure 2]. The most frequently 
Strohmeier et al.: How to improve organisational staff support?

voiced suggestions by both national and international staff related to improved access to psychosocial support services (sub-theme 1.1). Responses under this sub-theme showed that humanitarian workers consider the provision of additional, high-quality services as well as continuous access to services during and after deployment as important. The responses also revealed that participants seek especially support in dealing with daily stress in comparison to the traditional approach of acute stress interventions. One national staff phrased it as follows:

'Psychosocial support during assignment for on-going persistent stress associated with the environment, not only when/before an acute incident occurs' (male national staff, NNGO).

Regular and more frequent, paid time off in the form of leave and especially $R \& R$ was also repeatedly suggested, especially by international staff (sub-theme 1.2). Most of these suggestions referred to the shortening of existing R\&R cycles, for example to 10 , eight and four weeks. Responses showed that 'work-life balance should be emphasised' by organisations and revealed the need to

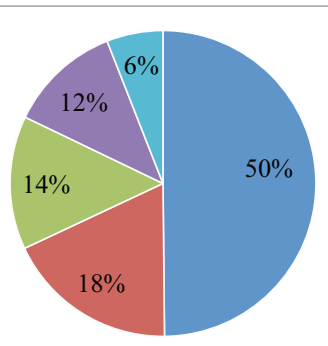

- Theme 1: Competitive salary and benefit packages

- Theme 2: Internal work climate and organisational culture

- Theme 3: Equality within and between organisations

- Theme 4: Skill enhancement and personal development

- Theme 5: Physical safety and security

Figure 1: Share of humanitarian workers' suggestions per theme ensure breaks are indeed time free of work, for instance through the 'requirement to leave laptops when going for leave or R\&R'. Participants also communicated the urgency to make sure staff take time off for their own good and that of their colleagues:

'Make sure that $R \& R$ is in place and is respected. The staff in worse condition are often the ones who do not want to go and who create more problems and stress for the rest of the team' (male international staff, INGO).

A comparatively large number of participants, the majority of these being national staff, suggested the increase of salaries (sub-theme 1.3). Most of the participants did not elaborate on the background of their request. Only one participant contextualised his suggestion by referring to the changing economic conditions in South Sudan:

'Review salaries (this is because some of the salary policies are last reviewed since 2008 when economic situation were better and the local currency were stronger but now local currency is getting weaker and weaker every day thus national staff salaries cannot keep up with economic hardship due to skyrocketing daily commodity prices in market)' (male national staff, INGO).

A similar share of suggestions referred to more staff support in generic terms (sub-theme 1.4). These requests for 'general staff welfare' were vague and did not include precise information on the type of services to be provided.

Improving access to medical services was of particular relevance for national staff (sub-theme 1.5). Suggestions under this sub-theme referred especially to 'regular health and mental check-ups' and the provision of drugs. One participant expressed the need for preventive measures in form of vaccines as protection from common diseases:

'Provide mandatory vaccination for hepatitis, which is very common and a great emotional drain on unsuspecting international staff who contract it' (male international staff, INGO).

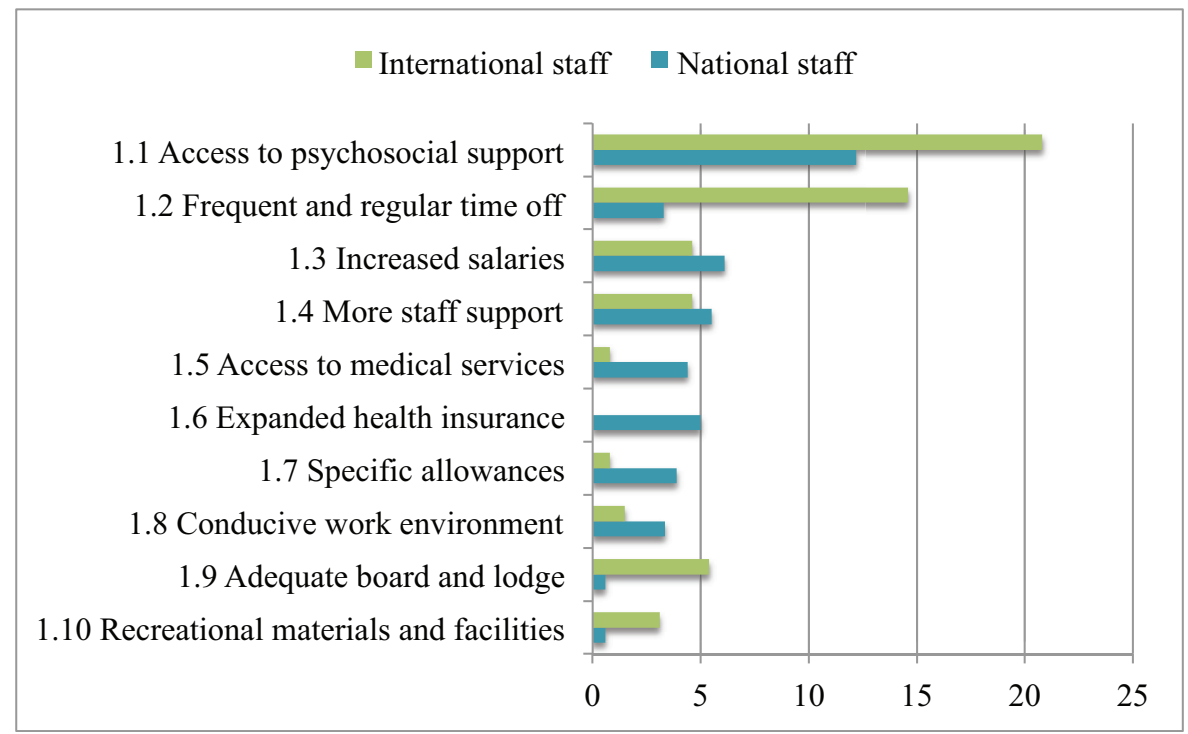

Figure 2: Share of national and international staff's suggestions related to competitive benefits and salary packages (\%) 
Strohmeier et al.: How to improve organisational staff support?

To utilise (additional) medical services and facilitate access for their family members, some national staff requested the expansion of health insurance schemes (sub-theme 1.6). As one national staff stated, staff welfare should always be a priority, 'especially full medical insurance for employees and their registered dependents'.

Some national staff also suggested the introduction of specific allowances (sub-theme 1.7). These referred largely to 'danger pay especially in war zone areas' or 'hardship allowances catering for the risks staff are undergoing'.

A few participants referred to the importance of conducive work environments (sub-theme 1.8). Most of them were vague as to what they meant with 'conducive' in this context. The two national staff who were more precise referenced 'communication equipment' and equipment such as 'quick run bags, transportation and communication'.

Mainly international staff suggested improvements towards an adequate boarding and lodging (sub-theme 1.9) but rarely specified their requests. However, one participant highlighted the need to consider gender:

'Female only accommodation areas within compounds (i.e. the large UNMISS base). Women should always have access to a bathroom close to their accommodation' (female international staff, UN).

A comparatively small share of the participants suggested the provision of recreational materials and facilities (sub-theme 1.10). This included improvements of social areas and the provision of games, such as 'balls, nets, indoor games'.

\section{Theme 2: Internal work climate and organisational culture}

The second theme that emerged referred to the internal work climate and culture of organisations. It emerged out of six sub-themes [Figure 3]. Participants, especially international staff, considered enhancing team cohesion of particular importance in this context (sub-theme 2.1). They voiced their wishes 'to get together in a team to share experiences once in a while', yet remained largely unspecific regarding the frequency of these gatherings. Merely one international staff recommended 'monthly meetings', and one national staff suggested 'a get together for all staff at least twice a year', an initiative that does currently not exist in his organisation.

The need for improved leadership and cooperation with management emerged as another sub-theme (sub-theme 2.2). The urgency for better communication voiced by multiple participants becomes evident in the following response:

'The managers should be supportive of staff but not always shouting at them in case of any mistakes done by staff. The management should listen to staff's complaints and ensure that it is handled to allow staff to concentrate on his or her work' (male national staff, NNGO).

Related to the above are suggestions about motivational support (sub-theme 2.3). National staff exclusively voiced these. Although most of them did not elaborate why this was necessary and how this need could be met, one participant suggested 'exposure to other working environments in other countries the agency works in' as a motivator. Another one referred to financial incentives.

Some participants suggested the development of separate staff support policies and the integration of staff support services in organisational processes (sub-theme 2.4). This included a strong statement on the funding of staff welfare:

'Stop considering people a number; put some resources into welfare of staff' (male international staff, UN).

International staff exclusively suggested improving the current hiring practices (sub-theme 2.5). They highlighted the urgency to appoint staff able to perform the tasks incumbent upon their positions, and the hiring of additional staff. Two of these accounts were exceptionally elaborate and personal:

'Ensure that the hired staff is up to the position they are covering. Being in a particularly hard context many NGOs are 'forced' to hire not qualified staff also for key positions. The consequences of these choices are oftentimes terrible (for beneficiaries and colleagues) and almost never reported' (male international staff, INGO).

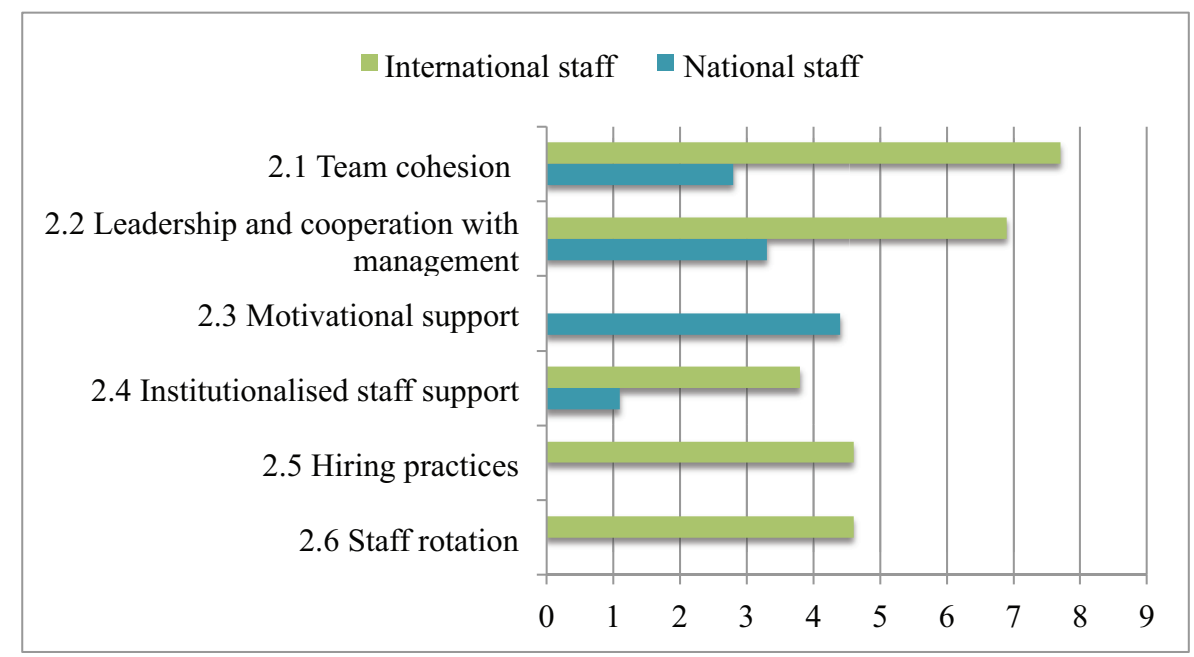

Figure 3: Share of national and international staff's suggestions related to internal work climate and organisational culture (\%) 
Strohmeier et al.: How to improve organisational staff support?

'Agencies should hire additional staff to decrease the often highly demanding and unhealthy workload, which is in my case one of my biggest sources of stress and frustration, resulting in a constant feeling of drowning with no end in sight' (female international staff, INGO).

Facilitating staff rotation, especially the rotation from hardship to safer duty stations, was another suggestion from international staff (sub-theme 2.6). Some participants provided specific information on rotation timelines, such as 'every two to three years', and some established an association between rotation and work climate, including a focus on those in high-level positions:

'Managers need change. If they stay long some may become a burden to others' (male international staff, INGO).

\section{Theme 3: Equality within and between organisations}

This theme emerged from five sub-themes, and especially national staff's suggestions included references to equality within and between organisations [Figure 4].

Many suggestions focused on inequalities between employees and the need to address these in generic terms (sub-theme 3.1). These included that 'national and international staff should be treated equally' and 'with equal rights and humanity'.

In addition to the individual level, some participants suggested addressing inequalities on the organisational level (sub-theme 3.2). The focus thereby was especially on (national) NGOs and the scaling up of their services. This becomes evident in the following statement:

'National organisations in particular need to focus more on support services for their staff to also help in the quality of work they are implementing' (male national staff, NNGO).

Mainly national staff highlighted the need to alleviate differences in salaries specifically between national and international staff (sub-theme 3.3). Particularly, they requested higher salaries, and one participant explained why:

'Treat both the national and international staff equally in terms of gross pay by at least giving the nationals half the pay international staff get. It is not fair to give international staff 500 USD and national staff 300 SSP [South Sudanese Pounds; in April 2017 an approximate conversion value of 3 USD (Trading Economics, 2018)] per day for fieldwork' (male national staff, INGO).

$\mathrm{R} \& \mathrm{R}$ emerged as another distinct area where mainly national staff voiced the need to address persisting inequalities (sub-theme 3.4). They emphasised the importance of $\mathrm{R} \& \mathrm{R}$ 'for all humanitarian workers whether international or local'.

Lastly, some national staff requested changes in organisations' evacuation policies of the organisations towards equal treatment of national and international staff (sub-theme 3.5). They worried about the high level of risk in field environments and being left behind by their organisations, which 'exposes them to danger of losing their lives'.

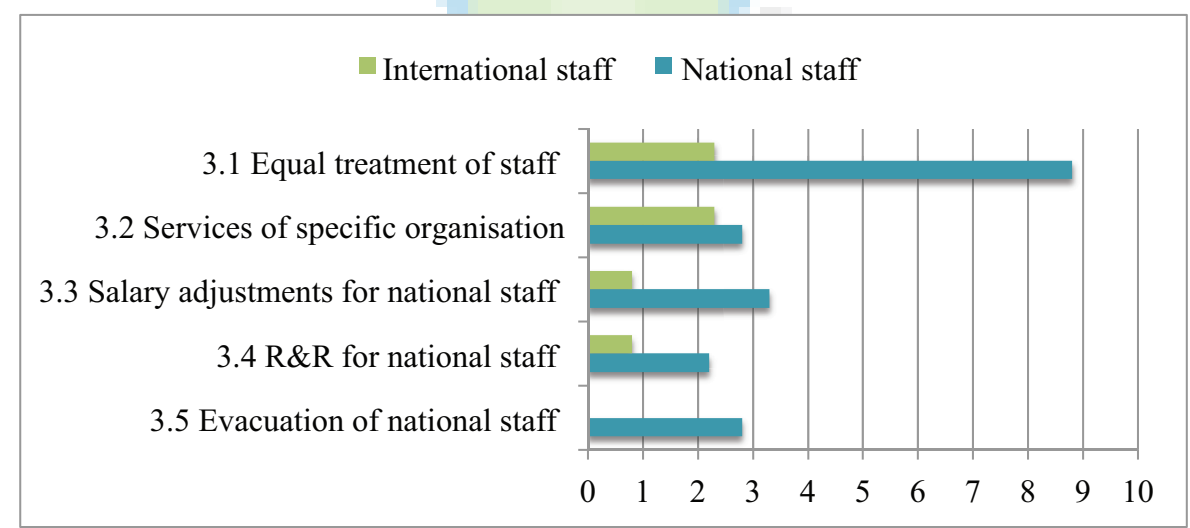

Figure 4: Share of national and international staff's suggestions related to equality within and between organisations (\%)

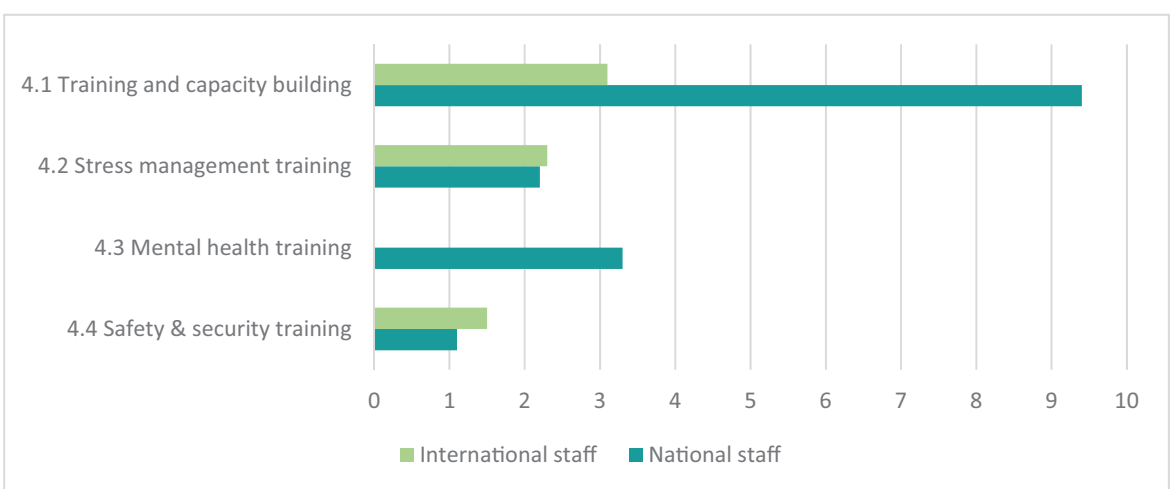

Figure 5: Share of national and international staff's suggestions related to skill enhancement and personal development (\%) 
Strohmeier et al.: How to improve organisational staff support?

\section{Theme 4: Skill enhancement and personal development}

This theme emerged from four sub-themes related to skill enhancement and personal development [Figure 5]. Particularly national staff considered training and capacity building important (sub-theme 4.1). Although some specified staff or employees as the target audience, most did not clarify the areas in which training and capacity building were needed. They generically suggested for organisations to 'send their staff for training'.

A smaller fraction of suggestions was more precise and facilitated the formulation of three distinct sub-themes on training content. One of these refers to training in stress management (sub-theme 4.2). As one national staff put it, 'training should be offered monthly especially in South Sudan for stress management for all staff'. Other suggestions referred specifically to training in the area of mental health (sub-theme 4.3). National staff exclusively requested this. Lastly, some participants suggested focused training on safety and security (sub-theme 4.4). This included training 'on safety measures in the geographical area they work in in South Sudan' and on typical issues, such as 'how to manage convoy attacks'.

\section{Theme 5: Physical safety and security}

Only few suggestions included references to physical safety and security, and national staff delivered the majority of these [Figure 6].

Most suggestions were similar and only two sub-themes emerged. The first sub-theme collates statements that highlighted the need to prioritise staff safety (sub-theme 5.1). The vast majority of these was vague and did not specify distinct threats and how to address these, respectively. Only one participant mentioned a specific security threat, sexual harassment, and offered advice how to address this situation:

'Enforce stricter harassment policies; whilst so far I am not aware that this is an issue in my organisation, there is certainly a culture of sexual harassment by fellow aid workers' (female international staff, INGO).
The same participant also reflected in detail about her personal experience with regards to staff safety and the lack thereof, respectively:

'One of my biggest stress factors is the very volatile security environment. Whilst I accept the risks of this job, I feel my organisation does not provide adequate security management policies, and consequently does not fully adhere to its duty of care. The issue has been raised and senior management are aware, but I feel no action is being taken, despite complaints from many different staff' (female international staff, INGO).

The second sub-theme collates suggestions for collaboration between the organisations to improve safety and security (sub-theme 2.2). Again, most of the suggestions did not provide details on such cooperation. Only two participants were more precise and suggested the UN Mission in South Sudan and the UN Police to take the lead, including ensuring that staff based in field locations 'can feel protected during their working time and (...) free to deal directly with beneficiaries'.

\section{Discussion}

It stands out that participants' suggestions capture a wide range of themes. With regards to the relative prominence of the five themes, we anticipated more suggestions related to safety and security. The limited attention to this component of staff support in the precarious context of South Sudan may be linked to the complex socio-psychological components and processes involved in risk perception and decisionmaking, such as reliance on incomplete data, risk communication, individual memories and habits, and unconscious emotional processes (Gee \& Skovdal, 2017; Gent, 2002). At the same time, at least international staff's engagement in the sector is in essence voluntary risk taking. The absence of references to safety and security can thus also be interpreted as the acceptance of risk as integral element of the job. Although motivations to join and stay in the sector are manifold (Ager \& Iacovou, 2014; Arcaro, 2016), this supports the argument of Roth (2015) to consider humanitarian workers as 'edgeworkers' (Lyng, 1990) - people seeking risky activities instead of avoiding them.

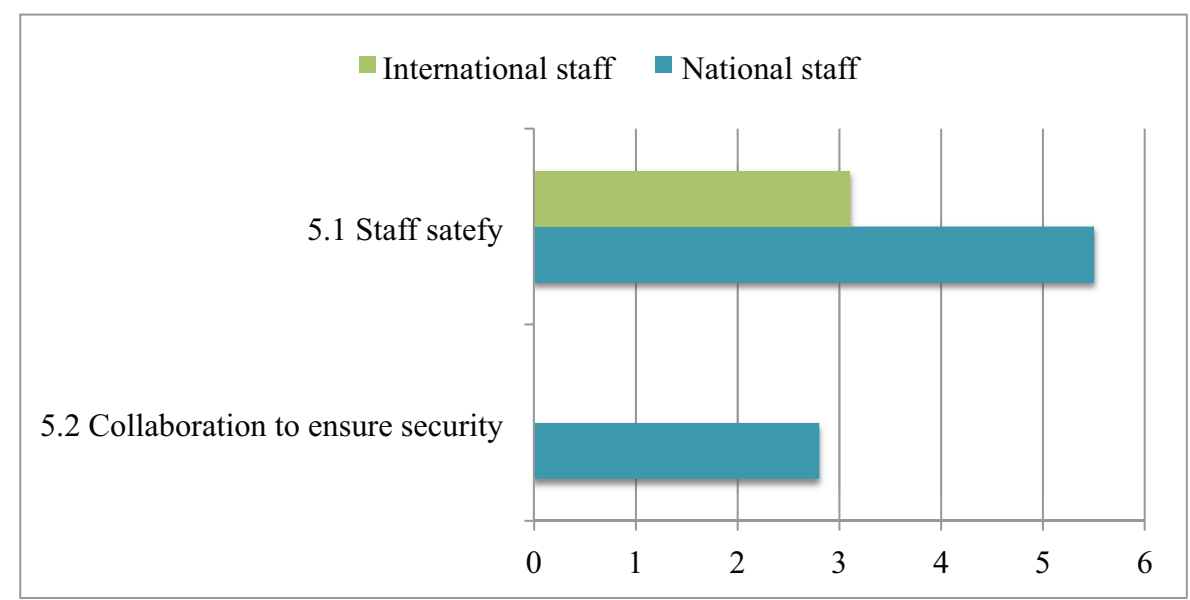

Figure 6: Share of national and international staff's suggestions related to physical safety and security (\%) 
Strohmeier et al.: How to improve organisational staff support?

That both national and international staff requested especially improved access to psychosocial support services deviates somewhat from earlier research (Welton-Mitchell, 2013). This finding is particularly noteworthy with regards to national staff, given the significant stigma attached to mental illness in the South Sudanese society. There are differences in the perception of common, temporary physical and emotional reactions to trauma and chronic stress, and severe, persisting consequences. Despite this, the understanding of mental health in the country is limited overall, and support and/or treatment is uncommon including due to the lack of public mental health and psychosocial support services (Amnesty International, 2016; IRIN, 2012). It is likely that working in the humanitarian sector and the exposure to the mental health and psychosocial support programmes offered by organisations (even if to beneficiaries only) had a sensitising effect on national staff and raised their awareness of the benefits of these.

Robust research on the efficacy of mental health and psychosocial support services among humanitarian workers in general and South Sudan in particular is scarce, and a better evidence base is needed to identify appropriate, context-specific measures. However, evidence from other crises shows a beneficial impact (Fouchier \& Kedia, 2018), and multiple studies on humanitarian workers found an association between chronic stress exposure and mental health problems (e.g., Ager et al., 2012; Lopes-Cardozo et al., 2012). This suggests that services promoting work-life balance and stress management may be particularly useful and match humanitarian workers' requests for training in stress management, mental health, and safety and security. Access to mental health and psychosocial support is also in line with the most prominent staff support guidelines (Antares Foundation, 2012; IASC, 2007) and a component of organisations' duty of care (IASC, 2007; Kruijk, 2014). Judged on this basis, organisations' investment in such services is not only smart but also a necessary action.

As expected, and apart from psychosocial support, top priorities of national and international staff for staff support differed greatly. The fact that many national staff called for capacity building and training without further specification can likely be attributed to organisations' human resource management and the employment structure of the sector. The vast majority of national staff who participated in the survey had a first university degree (BA) accompanied by the competitive advantage of being familiar with the local context. However, the majority of job options in the sector available to national staff are general service positions or lower grade professional jobs, oftentimes linked to the direct assistance of beneficiaries (Stoddard et al., 2011). Many national staff experience difficulties in proving their professionalism in the absence of additional certificates and references and moving up the career ladder, including switching to international positions. Even with additional training, career change is not guaranteed, and national staff's newly acquired skills oftentimes remain underutilised (IRIN, 2013). Restricted career opportunities can lead to increased stress and further setbacks in motivation and thus, impact the mental health and wellbeing of staff (Lopes Cardozo et al., 2005). Supporting career progression, for instance through needs-based training, supervision and feedback, and individual development plans, is hence beneficial for staff and organisations.

In addition to their frequent yet generic call for equal treatment, numerous national staff requested increases in salaries. Differences in remuneration between national and international staff constitute a major and recurring focus of discussion in the literature on humanitarian work (e.g., Canavera, 2016; Denskus, 2017; McWha-Hermann, Jandric, Wakefield, Carr, Grund, Moutou, 2017; Pauletto, 2018). Research from McWha-Hermann et al. (2017) on fairness in aid remuneration shows that dual salary systems contribute to feelings of injustice and demotivation. However, the topic is complex and raises additional questions, including if suggestions related to salaries fall under the realm of staff support: whereas our data show that humanitarians equate remuneration with staff support, none of the most prominent guidelines on staff support (Antares Foundation, 2012; IASC, 2007) refer to salaries or the adjustment thereof in any way. In light of this, further efforts towards achieving a unified understanding of staff support are essential. Irrespective of this and given the demonstrated significance of the debate, increased attention of organisations, for instance through clear, nuanced discourse and information related to dual payment schemes as well as periodic pay analyses, would present a desirable development.

The high levels of stress associated with humanitarian work and the geographical distance between duty station, home country and whereabouts of family members and long-established social networks are plausible explanations for international staff's frequently voiced need for time off. Many humanitarians communicate dissatisfaction regarding high work demands and extended stays in unstable environments, which are often at odds with their desire to maintain relationships. This situation - operating in a work intensive, multi-cultural context away from personal support networks - offers also a strong explanation why especially international staff requested services towards enhancing team cohesion: whereas national staff may have resettled their immediate families to safer places in neighbouring countries, they generally still have options to socialise outside work. For international staff in field environments, however, it is mainly colleagues who are available to take on roles to fulfil needs for social support and companionship, thereby becoming 'newly constructed, fictive families' (Thomas, 2011).

Although some initiatives can be implemented at low cost, adjusting existing and establishing new staff support services is subject to funding. However, organisations in South Sudan currently vastly lack financial resources available for staff care (Strohmeier, 2018). To facilitate change, additional awareness raising and evidence-based lobbying with donors accompanied by adequate internal budgeting, including through allocation of core and project funds to staff support, are thus essential. 
Strohmeier et al.: How to improve organisational staff support?

\section{Limitations}

Our study has limitations. The survey topic - mental health and wellbeing - may have influenced the suggestions of participants. The design did not provide the opportunity to follow-up with participants and probe their answers. Some organisations did not respond or declined our request to support the survey, and the response rate was low [but in line with comparable research using an online survey (Niland, Polastro, Donini, \& Lee, 2015)]. Language barriers and restricted Internet access likely prevented some humanitarian workers from participation, particularly those in remote field locations, which are associated with greater hardship than the duty station of Juba. The sample of respondents likely over/under-represented some segments of the humanitarian community in South Sudan, such as national staff who usually make up the majority of humanitarian workers in field locations (Stoddard et al., 2011). These limitations impact the transferability of results.

\section{Conclusion}

Our study allows for drawing the following conclusions: first, humanitarian workers in South Sudan want to engage in discussions on staff support and hold views on how to improve the current services. This offers an opportunity for organisations to inform service provision and present a starting point for identifying tangible actions. Second, the vast majority of suggestions from both national and international staff referred to improved access to psychosocial support services, making it a clear case for organisations to assess their services in this area. This should capture research on locally meaningful practices to ensure efficacy. Third, the differences in priorities of national and international staff indicate the need to adjust systems to do better justice to the humanitarian community as a whole. However, given the breadth of suggestions and the difference between these and recommendations reflected in prominent staff support guidelines, further efforts towards achieving a unified understanding of staff support are needed to manage expectations of staff and holding organisations accountable.

\section{Acknowledgements}

We thank all humanitarian organisations in South Sudan that supported the online survey on the mental health and wellbeing of humanitarian workers, especially the designated focal points within organisations who facilitated the smooth implementation of the survey. We also thank all humanitarian workers who participated in the survey for taking the time and sharing their views with us. We highly appreciate the valuable comments from Professor Catherine Panter-Brick that greatly improved this manuscript.

\section{Financial support and sponsorship}

The study was supported by the German-American Fulbright Commission, the Santander Universities Research Grant Fund and the The Whitney and Betty MacMillan Center for International Affairs and Area Studies at Yale.

\section{Conflicts of interest}

There are no conflicts of interest.

\section{ReFERENCES}

Ager, A., \& Iacovou, M. (2014). The co-construction of medical humanitarianism: Analysis of personal, organizationally condoned narratives from an agency website. Social Science \& Medicine, 120, 430-438. doi:http://dx.doi.org/10.1016/j.socscimed.2014.05.053

Ager, A., Pasha, E., Yu, G., Duke, T., Eriksson, C., \& Cardozo, B. L. (2012). Stress, mental health, and burnout in national humanitarian aid workers in Gulu, Northern Uganda. Journal of Traumatic Stress, 25, 713-720. doi:10.1002/jts.21764

Amnesty International. (2016). Our hearts have gone dark: The mental health impact of South Sudan's conflict. London:Amnesty International.

Antares Foundation. (2012). Managing stress in humanitarian workers: Guidelines for good practice (3rd ed.). Amsterdam:Antares Foundation.

Arcaro, T. (2016). Escapism or altruism: What really motivates aid workers? Retrieved from http://www.theguardian.com/global-development-professionals-network/2016/apr/29/what-keeps-aid-workers-humanitarians-in-the-job

Bengtsson, M. (2016). How to plan and perform a qualitative study using content analysis. NursingPlus Open, 2, 8-14. doi:https://doi.org/ 10.1016/j.npls.2016.01.001

Brunello, N., Davidson, J. R. T., Deahl, M., Kessler, R. C., Mendlewicz, J., Racagni, G., \& Zohar, J. (2001). Posttraumatic stress disorder: Diagnosis and epidemiology, comorbidity and social consequences, biology and treatment. Neuropsychobiology, 43, 150-162. doi:10.1159/000054884

Canavera, M. (2016). The local/international divide: Reflection of a deeper problem in humanitarian aid? Retrieved from http://www. huffingtonpost.com/mark-canavera/the-localinternationaldi_b_9247834.html

Care for the caretakers. (2010). Care for the caretakers: Rolling out a protocol or developing tailor-made programmes on the spot? Intervention, 8(2), 165-169.

Curling, P., \& Simmons, K. B. (2010). Stress and staff support strategies for international aid work. Intervention, 8(2), 93-105.

Denskus, T. (2017). The salary gap between expat and local aid workersit's complicated. Working in development. Retrieved from http:// www.theguardian.com/global-development-professionals-network/ 2017/apr/19/the-salary-gap-between-expat-and-local-aid-workersits-complicated

Deutsche Welle. (2016). Embassies evacuate as violence worsens in South Sudan. Retrieved from http://www.dw.com/en/embassiesevacuate-as-violence-worsens-in-south-sudan/a-19391966

Elo, S., \& Kyngas, H. (2007). The qualitative content analysis process. Journal of Advanced Nursing, 62(1), 107-115. doi:10.1111/j.13652648.2007.04569.x

Eriksson, C. B., Cardozo, B. L., Foy, D. W., Sabin, M., Ager, A., Snider, L. . . Simon, W. (2012). Predeployment mental health and trauma exposure of expatriate humanitarian aid workers: Risk and resilience factors. Traumatology, 19(1), 41-48. doi:10.1177/1534765612441978

Fouchier, C. D., \& Kedia, M. S. (2018). Trauma-related mental health problems and effectiveness of a stress management group in national humanitarian workers in the Central African Republic. Intervention, 16(2), 103-109. doi:10.4103/INTV.INTV_9_18

Gee, S., \& Skovdal, M. (2017). The role of risk perception in willingness to respond to the 2014-2016 West African Ebola outbreak: A qualitative study of international health care workers. Global Health Research and Policy, 2, 21. doi:https://doi.org/10.1186/s41256-017-0042-y.

Gent, M. (2002). Weighing up the risks in aid work. Humanitarian Exchange, 21, 17-19

Gilbert, J. (2016). 'The problem is the silence': Challenges providing support to local INGO staff in Gaza. Intervention, 14(1), 60-69.

Graneheim, U. H., \& Lundman, B. (2004). Qualitative content analysis in nursing research: Concepts, procedures and measures to achieve trustworthiness. Nurse Education Today, 4, 105-112. doi:10.1016/ j.nedt.2003.10.001

Humanitarian Outcomes. (2016). Aid worker security report 2015: Figures at a glance. London: Humanitarian Outcomes.

Humanitarian Outcomes. (2017). Aid worker security report 2016 : Figures at a glance. London: Humanitarian Outcomes.

IASC. (2007). IASC Guidelines on Mental Health and Psychosocial Support in Emergency Settings. Geneva: IASC. 
Strohmeier et al.: How to improve organisational staff support?

ICSC. (2016). A guide to the mobility and hardship scheme and related arrangements. n.p.: United Nations.

ICSC. (2017). Hardship classification: Consolidated list of entitlements circular. n.p.: United Nations.

IRIN. (2012). Urgent need for mental healthcare. Retrieved from http:// www.irinnews.org/news/2012/08/27/urgent-need-mental-healthcare

IRIN. (2013). Helping local aid workers build meaningful careers. Retrieved from https://reliefweb.int/report/world/helping-local-aidworkers-build-meaningful-careers

Kahn, S. (2018). 'Our NGO family has suffered a tragedy, and we will survive.' Evaluating a crisis response intervention with expatriate aid workers in Afghanistan. Intervention, 16(1), 38-45. doi:10.1097/WTF. 0000000000000154

Kruijk, E. v. d. (2014). Duty of care in humanitarian crises. Retrieved from http://www.centreforsafety.org/news/duty-of-care-in-humanitarian-crises- $/$

Lopes Cardozo, B., Gotway Crawford, C., Eriksson, C., Zhu, J., Sabin, M., Ager, A., \& Simon, W. (2012). Psychological distress, depression, anxiety, and burnout among international humanitarian aid workers: A longitudinal study. PLoS ONE, 7(9), e44948. doi:10.1371/journal.pone.0044948

Lopes Cardozo, B., Holtz, T. H., Kaiser, R., Gotway, C. A., Ghitis, F., Toomey, E., \& Salama, P. (2005). The mental health of expatriate and Kosovar Albanian humanitarian aid workers. Disasters, 29(2), 152-170. doi:10.1111/j.0361-3666.2005.00278.x

Lopes Cardozo, B., Sivilli, T. I., Crawford, C. A., Scholte, W. F., Petit, P., Ghitis, F., \& Eriksson, C. (2013). Factors affecting mental health of local staff working in the Vanni region, Sri Lanka. Psychological Trauma: Theory, Research, Practice, and Policy, 5(6), 581-590. doi:10.1037/a0030969

Lyng, S. (1990). Edgework: A social psychological analysis of voluntary risk taking. American Journal of Sociology, 95(4), 851-886.

McKay, L. (2011). Resilience: Building resilient managers in humanitarian organizations. Strenghtening key organizational structures and personal skills that promote resilience in challenging environments. n.p.: People in Aid.

McWha-Hermann, I., Jandric, J., Wakefield, S., Carr, S. C., Grund, C., \& Moutou, M. (2017). Project FAIR: Exploring practical pathways for reward fairness in international NGOs. Edinburgh: University of Edinburgh.

Niland, N., Polastro, R., Donini, A., \& Lee, A. (2015). Independent whole of system review of protection in the context of humanitarian action. n.p.: Norwegian Refugee Council.

Omidian, P., \& Panter-Brick, C. (2015). Dignity under extreme duress: The moral and emotional landscape of local humanitarian workers in the Afghan-Pakistan border areas. In S. Abramowitz, C. PanterBrick, \& P. Piot (Eds.), Medical humanitarianism: Ethnographies of Practice. (pp 23-40) Philadelphia: University of Pennsylvania Press.
Pauletto, E. (2018). The shocking enequity in foreign aid nobody talks about. Retrieved from https://brightthemag.com/inequity-foreignaid-workers-security-pay-gap-international-humanitarian62ae974cca3

Pigni, A. (2014). Building resilience and preventing burnout among aid workers in Palestine: A personal account of mindfulness based staff care. Intervention, 12(2), 231-239.

Porter, B., \& Emmens, B. (2009). Approaches to staff care in international NGOs. London: People in Aid/InterHealth.

Roth, S. (2015). Aid work as edgework - Voluntary risk-taking and security in humanitarian assistance, development and human rights work. Journal of Risk Research, 18(2), 139-155. doi:https://doi.org/ 10.1080/13669877.2013.875934

Shah, S. A., Garland, E., \& Katz, C. (2007). Secondary traumatic stress: Prevalence in humanitarian aid workers in India. Traumatology, 13 (1), 59-70.

Stoddard, A., Harmer, A., \& Haver, K. (2011). Safety and security for national humanitarian workers. New York: UNOCHA.

Strohmeier, H. (2018). Organizational staff support in South Sudan: An overview of current services and recommendations for future initiatives. Edinburgh: Queen Margaret University, Antares Foundation.

Strohmeier, H., \& Scholte, W. F. (2015). Trauma-related mental health problems among national humanitarian staff: A systematic review of the literature. European Journal of Psychotraumatology, 6, 28541. doi:10.3402/ejpt.v6.28541

Surya, M., Jaff, D., Stilwell, B., \& Schubert, J. (2017). The importance of mental well-being for health professionals during complex emergencies: It is time we take it seriously. Global Health: Science and Practice, 5(2), 188-196. doi:10.9745/GHSP-D-17-00017

Thomas, R. (2011). Managing transition sbetween 'field' \& 'home': Facing the psychological impact of humanitarian crisis. In A. Vautravers, \& L. Werner (Eds.), Psychological impact of humanitarian crises: 14th humanitarian conference (pp 226-245). Webster University.

Trading Economics. (2018). South Sudanese pound. Retrieved from https://tradingeconomics.com/south-sudan/currency

UN News. (2017). UN aid chief urges global action as starvation, famine loom for 20 million across four countries. https://news.un.org/en/ story/2017/03/553152-un-aid-chief-urges-global-action-starvationfamine-loom-20-million-across-four

UNHCR. (n.d.).South Sudan refugee crisis: Spiraling violence, conflict and severe food shortage have endangered millions of lives. Retrieved from http://www.unrefugees.org/emergencies/south-sudan/

UNOCHA South Sudan. (2017). Humanitarian response plan: January-December 2018. n.p.: UNOCHA.

UNOHRM. (n.d.). Non-family duty stations. Retrieved from http://www. un.org/Depts/OHRM/salaries_allowances/allowances/nonfam.htm

Welton-Mitchell, C. E. (2013). UNHCR's mental health and psychosocial support for staff. Geneva: UNHCR. 